CORRECTION

\title{
Correction: Artificial intelligence enabled parabolic response surface platform identifies ultra-rapid near-universal TB drug treatment regimens comprising approved drugs
}

\section{The PLOS ONE Staff}

The ORCID iD is missing for the corresponding author. The publisher apologizes for the error. Author Marcus A. Horwitz's ORCID iD is: 0000-0001-6525-7147 (https://orcid.org/ 0000-0001-6525-7147).

In Table 2, Prothionamide was mistakenly abbreviated "PRS" instead of "PRO" in the second column, "Screening Test". Please see the corrected Table 2 here.

f open ACcess

Citation: The PLOS ONEStaff (2019) Correction: Artificial intelligence enabled parabolic response surface platform identifies ultra-rapid nearuniversal TB drug treatment regimens comprising approved drugs. PLoS ONE 14(5): e0217670. https://doi.org/10.1371/journal.pone.0217670

Published: May 30, 2019

Copyright: ๑ 2019 The PLOS ONE Staff. This is an open access article distributed under the terms of the Creative Commons Attribution License, which permits unrestricted use, distribution, and reproduction in any medium, provided the original author and source are credited. 
Table 2. Screening of combinatorial drugs in macrophage model of $M$. tuberculosis infection.

\begin{tabular}{|c|c|c|c|c|c|c|}
\hline & Screening test & Iteration \# 1 & $\begin{array}{l}\text { Iteration } \\
\# 2\end{array}$ & $\begin{array}{l}\text { Iteration } \\
\# 3 \mathrm{~A}\end{array}$ & \begin{tabular}{|l} 
Iteration \\
$\# 3 \mathrm{~B}$ \\
\end{tabular} & $\begin{array}{l}\text { Iteration } \\
\# 3 \mathrm{C}\end{array}$ \\
\hline Effect levels ${ }^{\mathrm{a}}$ & 2 & 3 & 3 & 5 & 5 & 3 \\
\hline Test runs ${ }^{\mathrm{b}}$ & 150 & 102 & 155 & 75 & 75 & 82 \\
\hline \multirow[t]{15}{*}{ Drugs $^{c}$} & $\mathrm{AC}$ & AC & AC & $\mathrm{AC}$ & AC & AC \\
\hline & BDQ & BDQ & BDQ & BDQ & BDQ & BDQ \\
\hline & CFZ & CFZ & CFZ & CFZ & CFZ & CFZ \\
\hline & DLM & DLM & DLM & DLM & DLM & DLM \\
\hline & RIF & RIF & RIF & RIF & RIF & RIF \\
\hline & SQ109 & SQ109 & SQ109 & SQ109 & SQ109 & SQ109 \\
\hline & PA824 & PA824 & PA824 & PA824 & & PA824 \\
\hline & PZA & PZA & PZA & PZA & & \\
\hline & EMB & EMB & EMB & & & \\
\hline & PAS & PAS & & & & \\
\hline & PRO & PRO & & & & \\
\hline & CYS & & & & & \\
\hline & INH & & & & & \\
\hline & LZD & & & & & \\
\hline & MXF & & & & & \\
\hline
\end{tabular}

${ }^{a}$ Screening test was conducted at individual drug concentration that yielded $0 \%$ or $10 \%$ of the maximal inhibition to the IPTG-induced green fluorescence signal. Iteration \# 1 was conducted at individual drug concentration that gave $0 \%$ or $10 \%$ of the maximal inhibition level and one-half of the concentration that gave $10 \%$ of the maximal inhibition level. Iteration $\# 2$ was done at $0 \%$ or $15 \%$ effect levels and one-half of the concentration that gave $15 \%$ of the maximal inhibition level. Iterations \#3A and $3 \mathrm{~B}$ were conducted at $0 \%$ or $20 \%$ effect level and two-thirds, one-half or one-fourth of the concentration that gave $20 \%$ of the maximal inhibition level. Iteration \#3C was conducted at $0 \%$ or $15 \%$ effect levels and one-half of the concentration that gave $15 \%$ of the maximal inhibition level.

${ }^{\mathrm{b}}$ Number of combinatorial drug test runs in each experiment.

${ }^{c}$ List of drugs tested at screening and each stage of iterations.

https://doi.org/10.1371/journal.pone.0217670.t001

On the right side of Fig 1, Group O was mistakenly omitted from the alphabetical list of drug regimen definitions. Please see the corrected Fig 1 here.



\begin{tabular}{cl} 
Group & \multicolumn{1}{c}{ Treatment } \\
\cline { 2 - 2 } A & Sham control \\
B & Standard Regimen \\
C & PRS Regimen II \\
D & RFT, AC, DLM, SQ109 \\
E & RFT, AC, DLM, PA824 \\
F & RFT, AC, PA824, SQ109 \\
G & RFT, AC, BDQ, SQ109 \\
H & RFT, AC, CFZ, DLM \\
I & RFT, AC, BDQ, DLM \\
J & RFT, AC, BDQ, CFZ \\
K & RFT, DLM, PA824, SQ109 \\
L & CFZ, AC, BDQ, DLM \\
M & CFZ, AC, BDQ, PZA \\
N & CFZ, AC, DLM, PA824 \\
O & CFZ, BDQ, PA824, SQ109 \\
P & CFZ, BDQ, DLM, PZA \\
Q & CFZ, BDQ, DLM, PA824
\end{tabular}

Fig 1. In vivo short-term efficacy screen of experimental regimens identified in macrophage studies using the PRS platform. M. tuberculosis infected mice were sham treated (Treatment Group A) or treated with the Standard Regimen (Treatment Group B), PRS Regimen II (Treatment Group C) or one of the top 4-drug combinations (Treatment Groups D-Q) identified from macrophage screening using the PRS platform starting from a pool of 15 TB drugs 5 days per week for 3 weeks. Three days after the last treatment, mice were euthanized to determine bacterial number in the lung. Standard Regimen is comprised of INH, RIF, EMB and PZA at 25, 10, 100 and $150 \mathrm{mg} / \mathrm{kg}$, respectively. PRS Regimen II is comprised of CFZ, BDQ, PZA and EMB at 25, 30, 450 and 100 mg/kg, respectively. Drug doses used in the top 4-drug experimental regimens are as follows: $200-50 \mathrm{mg} / \mathrm{kg}$ for AC, $30 \mathrm{mg} / \mathrm{kg}$ for BDQ, $25 \mathrm{mg} / \mathrm{kg}$ for CFZ, $2.5 \mathrm{mg} / \mathrm{kg}$ for DLM, $100 \mathrm{mg} / \mathrm{kg}$ for PA824, $450 \mathrm{mg} / \mathrm{kg}$ for PZA, $10 \mathrm{mg} / \mathrm{kg}$ for RPT and $25 \mathrm{mg} / \mathrm{kg}$ for SQ109.

https://doi.org/10.1371/journal.pone.0217670.g001 


\section{PLOS $\mid$ ONE}

\section{Reference}

1. Clemens DL, Lee B-Y, Silva A, Dillon BJ, Masleša-Galić S, Nava S, et al. (2019) Artificial intelligence enabled parabolic response surface platform identifies ultra-rapid near-universal TB drug treatment regimens comprising approved drugs. PLoS ONE 14(5): e0215607. https://doi.org/10.1371/journal.pone. 0215607 PMID: 31075149 